\title{
ADAPTACIÓN DE LENTES DE CONTACTO EN 133 OJOS CON ASTIGMATISMO IRREGULAR
}

\section{CONTACT LENS FITTING IN 133 EYES WITH IRREGULAR ASTIGMATISM}

\author{
GALINDO-FERREIRO A ${ }^{1}$, GALINDO-ALONSO J ${ }^{2}$, SÁNCHEZ-TOCINO H³ ${ }^{3}$, PALENCIA-ERCILLA J ${ }^{4}$
}

\section{RESUMEN}

Objetivos: Estudiar la adaptación de lentes de contacto en 133 ojos con astigmatismos irregulares.

Material y métodos: Se realizó un estudio retrospectivo en 133 ojos con astigmatismos irregulares. El criterio de inclusión fue tener un astigmatismo irregular imposible de corregir con gafas o con lentes de contacto de diseño estandard. Se efectuó una exploración oftalmológica completa, incluyendo topografía con Eye-Sys 2000. Las variables que se tuvieron en cuenta fueron: refracción, agudeza visual antes y después de la adaptación, etiología y tipo de lente de contacto.

Resultados: De la muestra 50\% fueron mujeres y $50 \%$ hombres, $52 \%$ de los ojos fueron derechos y $46 \%$ izquierdos y con afectación bilateral un $67 \%$. El 78,2\% (110 casos) de los ojos tratados correspondieron a queratoconos, 4 traumatismos corneales, 9 infecciones corneales y 6 astigmatismos idiopáticos.

De las 133 lentes de contacto que se adaptaron 103 fueron lentes híbridas $\left(\right.$ Softperm $\left.^{\circledR}\right), 20$ sistemas piggy-back, 5 hidrofílicas gruesas (Queratosoft $\left.{ }^{\circledR}\right), 4$ hidrofílicas y 3 rígidas gas permeables. La agudeza visual (AV) previa media fue de 0.28 (DE 0,24) (rango 0,1-0,8). Después de la adaptación de la lente de contacto la agudeza visual fue de 0,81 (DE
Purpose: To study the adaptation of contact lens in a sample of 133 eyes with irregular astigmatism.

Methods: A retrospective study was made in 133 eyes with irregular astigmatism. The selection criterion was to obtain a sample population with irregular astigmatism that was unlikely to be corrected with spectacles or conventional contact lens. A complete ophthalmologic exploration which included a topography with the Eye-Sys 2000 corneal topographer was made was made. The variables analyzed in the study were: refraction, visual acuity before and after the correction, cause of the astigmatism and contact lens used.

Results: An equal number of women and men were enrolled in the study. The right eye was studied in $52 \%$ of cases, and the left eye in $46 \%$. Both eyes were affected in $67 \%$ of the subjects. The reason for the astigmatism was keratoconus in 110 eyes (78.2\%), and there were 4 corneal injuries, 9 ocular infections, and 6 idiopathic astigmatisms. Among the contact lens used in the study: in 103 eyes a hybrid lens (Softperm ${ }^{\circledR}$ ) was adapted, in 20 eyes a piggyback system, in 5 eyes a thick hydrophilic lens (Queratosoft ${ }^{\circledR}$ ), in 4 eyes a hydrophilic contact lens and in 3 cases a rigid gas permeable contact lens. The average visual acuity before the adaptation was 0.28 (SD

\footnotetext{
Recibido: 18/4/06. Aceptado: 30/10/07.

Correspondencia:

Alicia Galindo Ferreiro

C/. Francisco Zarandona, esc. derecha, 1. ${ }^{\circ} \mathrm{A}$

47003 Valladolid

España

E-mail: ali_galindo@yahoo.es
}

1 Doctor en Medicina. Complejo Hospitalario de Palencia.

2 Doctor en Física. Hospital Universitario Río Hortega.Valladolid.

3 Doctor en Medicina. Hospital Universitario Río Hortega. Valladolid.

4 Doctor en Medicina. Hospital Clínico Universitario. Valladolid.

Comunicación presentada en el LXXXI Congreso de la S.E.O. (Zaragoza 2005). 
0,23) (rango 0,1-1). Se encontraron diferencias significativas entre la AV pre/postratamiento con una mejoría visual de 0,53 (DE 0,28).

Conclusiones: Sólo a través del manejo de multitud de lentes de contacto por un oftalmólogo especializado se puede llegar a un buen resultado visual-confort en ojo con astigmatismo irregular.

Palabras clave: Astigmatismo irregular, queratocono, lentes de contacto, lentes híbridas, sistema piggy-back.
0.24 ) (range 0.1-0.8). After the use of the lens the average visual acuity was 0.81 (SD 0.23) (range 0.11). Statistically significant differences between the visual acuity before and after treatment were found, with an improvement of 0.53 (SD 0.28) obtained.

Conclusion: Only with experience using a large variety of non-conventional contact lens can a specialist contact lens ophthalmologist achieve a good result (Arch Soc Esp Oftalmol 2007; 82: 747-752).

Key words: Irregular astigmatism, keratoconus, contact lens, hybrid lens, piggy-back system.

\section{INTRODUCCIÓN}

El astigmatismo irregular es una causa de pérdida de visión. Su corrección con lentes de contacto es el tratamiento de elección y la clave del éxito visual en la mayoría de los casos (1-3). El desafío es mantener al paciente con una tolerancia aceptable junto con una buena agudeza visual en córneas en continuo cambio (4).

Este estudio evalúa las características de los pacientes con astigmatismos irregulares, las diferentes etiologías del astigmatismo y pretende demostrar la posibilidad de lograr una agudeza visual aceptable con la adaptación de lentes de contacto. Para ello pensamos que es necesario el manejo de gran variedad de modelos de lentes de contacto y dominio de los sistemas de adaptación.

\section{SUJETOS, MATERIAL Y MÉTODOS}

\section{Población de pacientes}

Se llevó a cabo un estudio retrospectivo sobre 133 ojos en 79 pacientes con astigmatismos irregulares de todas las etiologías recogidos en nuestra Clínica Oftalmológica.

Como criterio de inclusión del estudio se admitieron todos los astigmatismos irregulares que no pudiera alcanzar con gafa o lente de contacto de diseño estandard una buena agudeza visual (AV).

\section{Datos recogidos}

Se cuantifican los siguientes parámetros: ojo (derecho/izquierdo), lateralidad (unilateral/bilate- ral), sexo, etiología del astigmatismo irregular, agudeza visual (AV) pre y post, refracción pre y postadaptación. Los tipos de lentes de contacto utilizadas fueron:

Grupo1: lente mixta [Softperm ${ }^{\circledR}$ (Ciba vision)].

Grupo 2: sistema piggy-back [Proclear ${ }^{\circledR}$ (Cooper vision)-Polycon II ${ }^{\circledR}$ (Lenticon), Proclear $^{\circledR}$-Permiflex $^{\circledR}$ (Eurolent s.1), Standard ${ }^{\circledR}$ (Ciba vision)-Permiflex $^{\circledR}$ (Eurolent s.l), Durasoft 3 color blends ${ }^{\circledR}$ (Ciba vision)-Polyfocal oxicon $100^{\circledR}$ (Lenticon), Standard ${ }^{\circledR}$ (Ciba vision)-Wolk A90 ${ }^{\circledR}$ (Eurolent), Standard ${ }^{\circledR}$ (Ciba vision)-Acrilens $\mathrm{I}^{\circledR}$ (Lentiflex), Standard ${ }^{\circledR}$ (Ciba vision)-Acrilens II tórica ${ }^{\circledR}$ (Lentiflex), Softmate $B^{\circledR}$ (Ciba vision)-Permiflex color ${ }^{\circledR}$ (Eurolent s.1), Softmate $B^{\circledR}$ (Ciba vision)-Acrilens II $^{\circledR}$ (Lentiflex)].

Grupo 3: lente hidrofílica gruesa [Queratosoft $38^{\circledR}$ (Lenticon)].

Grupo 4: lente hidrofílica [Zero 6 tórica ${ }^{\circledR}$ (Cooper vision), Zero 6 esférica $^{\circledR}$ (Cooper vision)].

Grupo 5: lente rígida gas permeable (RGP) [Keratocon policon ${ }^{\circledR}$ (Lenticon)].

Se ensayaron todas las combinaciones de lentes de contacto disponibles en nuestra consulta hasta que se obtuvo una correcta adaptación para cada paciente. La lente mixta utilizada se compone de un segmento central fabricado de pentasilcon y un ani1 lo periférico de material hidrofílico con $20 \%$ de agua. El diámetro del anillo central es de $8 \mathrm{~mm}$ y el total de 14,3 $\mathrm{mm}$ y de la zona óptica de $7 \mathrm{~mm}$. La lente hidrofílica gruesa es una lente con diseños tricurvos, espesor central aumentado (entre 0,4 y 0,6 $\mathrm{mm}$ ) y materiales de baja hidrofilia (glicerol en este caso) pueden ser una alternativa muy útil dentro de las LC hidrofílicas. El sistema piggyback o sándwich es la adaptación de una lente hidrofílica y encima una lente RGP. 
Se dividieron las causas del astigmatismo irregular en cinco grupos; queratocono, traumatismo corneal, leucoma por infección corneal, queratoplastia y astigmatismo irregular idiopático. Se entiende por astigmatismo irregular idiopático aquel astigmatismo que no tiene un patrón topográfico característico de queratocono y que no evoluciona en el tiempo. La refracción se obtuvo mediante un autorrefractómero automático objetivo y posteriormente se afinó la graduación mediante análisis subjetivo con los optotipos de la E Snellen, en las dos situaciones, preadaptación y posadaptación de la lente de contacto. Para la mejor comparación entre refracciones se calculó el equivalente esférico (EE) (igual a la potencia esférica de la lente más la mitad del astigmatismo).

\section{Procedimiento}

Se procedió a un examen oftalmológico completo al inicio de la adaptación y al mes de terminarla, en el que se incluía una topografía con el topógrafo EyeSys 2000 (EyeSys 2000 Corneal Analysis system. Eyesys Technologies, Inc. Houston. Texas. USA). El topógrafo y queratómetro nos fueron útiles para diagnosticar y tipificar el astigmatismo irregular, pero no para la elección del tipo de lente contacto.

Una vez etiquetado el astigmatismo irregular se comenzó la adaptación de una lente mixta, si ésta no se tolerara, a pesar de un buen ajuste, se probaría una lente hidrofílica gruesa. Si no se fabricaran parámetros adecuados o el astigmatismo residual fuera mayor de 1 dioptría recurriríamos a un sistema piggy-back. Solamente se utilizaron lentes RGP en último lugar o bien cuando el paciente no era capaz de realizar un parpadeo correcto que movilizara adecuadamente los procedimientos anteriores. El uso de la lente hidrofílica se reservó para astigmatismos irregulares de pequeña cuantía.

El tiempo de prueba de cada diseño y el tiempo de adaptación final fueron muy variables ya que en algunos casos se desecharon modelos en el primer momento y otras veces sólo cuando el paciente usaba las lentillas en su vida diaria. Una correcta adaptación se evaluó como un correcto centrado, movimiento y, en el caso necesario, un buen patrón de fluoresceína.

Se consideró éxito de la adaptación la comprobación de la tolerancia durante la jornada laboral (mínimo 8 horas) en un mes.

\section{RESULTADOS}

De los 133 ojos recogidos con astigmatismos irregulares se obtuvieron los siguientes datos. El $50 \%$ de los ojos pertenecían a hombres y el $50 \%$ a mujeres. Un $48 \%$ fueron ojos derechos y un $52 \%$ izquierdos. Un $33 \%$ de los pacientes tenían los dos ojos afectados y un $67 \%$ la patología era unilateral. En la figura 1 se puede comprobar que el grupo de edad con más afectados fue el comprendido entre 40-49 años (46 casos), seguida del grupo de 30-39 años (36 casos), y del 20-29 años con 31 casos. La edad media de diagnóstico del astigmatismo irregular en este estudio fue de 34,9 DE 10,7 años, siendo la edad máxima 70 años y la mínima 12 años.

Las diferentes etiologías vienen detalladas en la figura 2. La causa más frecuente de astigmatismo irregular fue el queratocono con 110 ojos seguida con nueve casos de astigmatismo causado por leucomas a causa de infecciones corneales, seis astig-

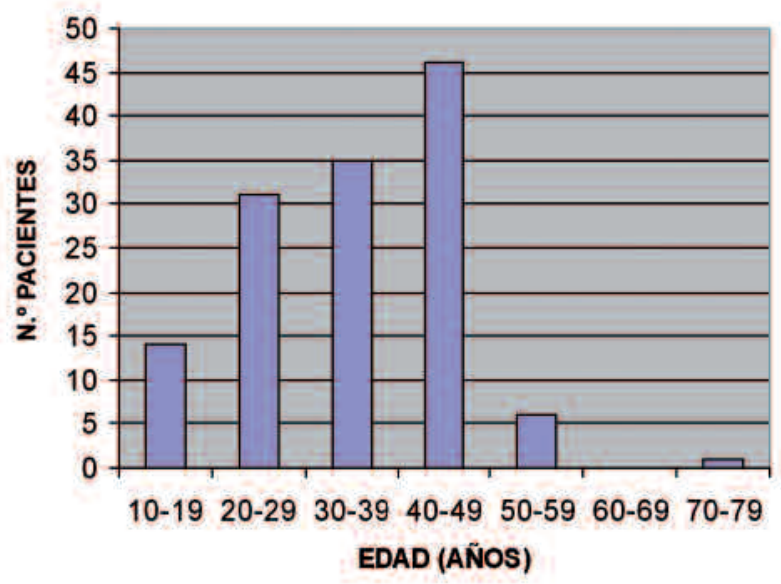

Fig. 1: Distribución de la muestra por edades.

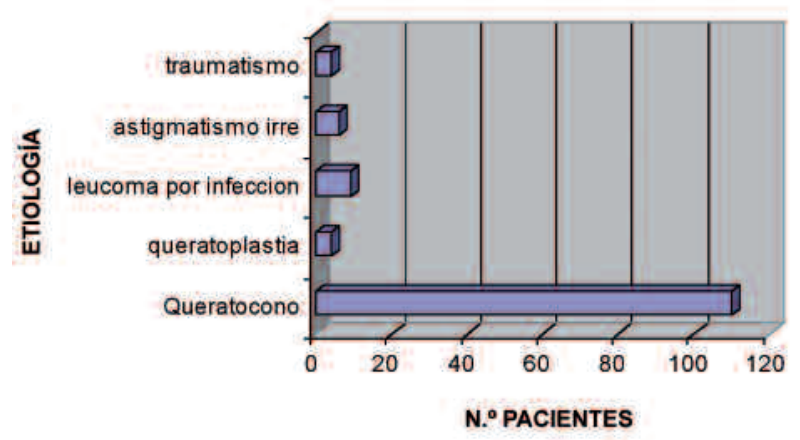

Fig. 2: Etiología del astigmatismo irregular. 
matismos irregulares idiopáticos, cuatro post-queratoplastias y cuatro traumatismos corneales.

Al cuantificar la refracción preadaptación de los ojos con astigmatismos irregular se detectó que un $60,9 \%$ de los casos fue imposible la realización de una refracción, por lo que sólo se pudo analizar en un $39,1 \%$ de los ojos. En este grupo se vio que el defecto de refracción, medido como EE, más frecuente fue el comprendido entre 0 y -8 dioptrías (Dp) (fig. 3) con un EE medio preadaptación de -5 (DE 4,3) Dp. Sin embargo, después de la adaptación de la lente de contacto en los 133 ojos se pudo realizar una correcta refracción del total detectando que el defecto de refracción más frecuente fue el grupos de -2 a -4 Dp (fig. 3) con un EE medio postadaptación de -2,5 (DE 4,5) Dp.

Como muestra la figura 4 la lente de contacto más utilizada fue la lente mixta (103 ojos) seguida de la piggy-back (17 ojos), hidrofílica gruesa (5 ojos), hidrofílica (4 ojos) y RPG en 4 ojos.

La mejor agudeza visual corregida (AV) preadaptación fue de 0,21 DE 0,24 mientras que la AV media post-adaptación fue de 0,81 (DE 0,23), con una mejoría visual media de 0,53 DE 0,28.

\section{DISCUSIÓN}

Estamos de acuerdo con Maguen (5) y Yeung (3) cuando señalan que la etiología más frecuente del astigmatismos irregular es el queratocono. En los estadios iniciales del queratocono, probablemente,

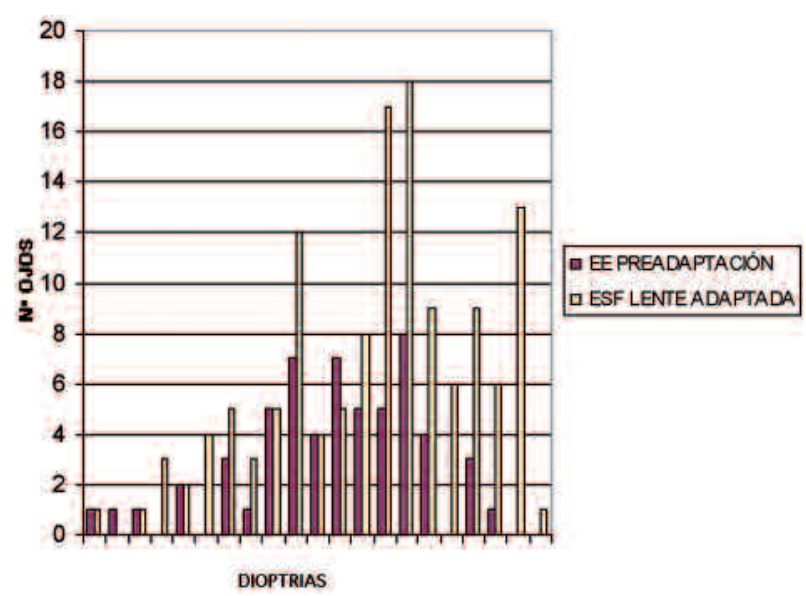

Fig. 3: Distribución del equivalente esférico (EE) en la muestra antes de la adaptación de la lente de contacto y postadaptación de la lente de contacto.

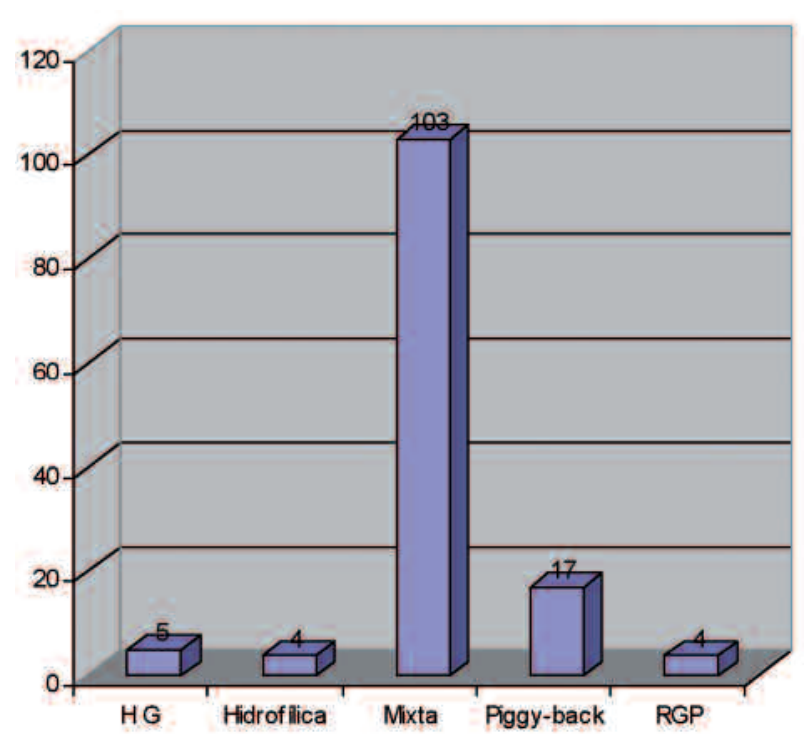

Fig. 4: Tipos de lentes de contacto adaptadas.

se pueda mejorar la AV con una gafa, con lentes de contacto hidrofílicas esféricas o bien tóricas, pero cuando esta enfermedad avanza, la córnea se hace más irregular y las correcciones ópticas comunes no logran mejorar la AV. Es en este momento cuando se debe pensar en comenzar la adaptación de otro tipo de lentes de contacto.

Aunque en este estudio no se han recogido datos de pacientes operados de cirugía refractiva, sabemos que el auge de esta técnica ha traído como consecuencia el hallazgo en el preoperatorio de queratoconos hasta entonces desconocidos, además de su efecto iatrogénico causante de astigmatismos irregulares.

Se consiguió adaptar una lente de contacto en cada uno de los 133 pacientes de forma que se logró una mejoría de la AV junto con una buena tolerancia. No se tuvo ninguna complicación grave en el periodo de adaptación. Hay que decir que no siempre ocurre así, porque dependiendo de la deformidad corneal hay ocasiones en las que nos es imposible la adaptación de una lente y se tendría entonces que plantear la posibilidad de cirugía.

La lente de contacto que se adaptó con más éxito (mejor AV-comfort) fue la lente de contacto mixta seguida por el sistema piggy-back. Según Yeung, Maguen, O’Donnell $(3,5,6)$ la lente de contacto que con más frecuencia se adapta en córneas irregulares es la RGP. En nuestra opinión, la RGP es un tipo de lente que funciona bien pero que produce en algunos casos incomodidad y en astigmatismos irregulares avanzados imposibilidad de adaptación. Debi- 
do a estos inconvenientes, a lo largo de nuestra experiencia, hemos tenido que ir buscando sustitutos a este tipo de lente para dar solución al problema visual de nuestros pacientes. Así, tanto la lente mixta como el sistema piggy-back al hacer que la córnea sea recubierta por una lente hidrofílica crean un mayor equilibrio en el movimiento y comfort para los pacientes a la vez que en su parte central se añade una lente rígida optimizando la visión.

Coincidiendo con Lim (5), se halló que la edad de diagnóstico de los astigmatismos irregulares estaba entre los 30-49 años. Hay que decir que es en este grupo de edades donde se diagnostica al paciente pero no debemos confundirnos con la edad de comienzo de la enfermedad, en el caso de que la causa del astigmatismo irregular sea el queratocono o el astigmatismo irregular idiopático, que como sabemos es difícil de conocer. Coincide la edad de comienzo y diagnóstico en las demás causas de astigmatismo irregular mencionadas en el estudio.

La corrección de un astigmatismo irregular es un desafío para el oftalmólogo. En la mayoría de los casos una gafa con la graduación adecuada no mejora la AV, dejando a las lentes de contacto como única opción de tratamiento antes de la cirugía. Por esta razón deben de agotarse todas las opciones en lentes de contacto en cada ojo con astigmatismo irregular. Sólo a través del manejo de multitud de tipos de lentes por un oftalmólogo especializado se puede llegar a un buen resultado visual en este tipo de pacientes tan complejos en los que el desafío es mantener una buena tolerancia junto con una adecuada AV en una córnea cambiante (4).

Como conclusión de nuestro estudio se puede decir que las lentes de contacto continúan teniendo un papel relevante en el tratamiento de los astigmatismos irregulares $(1,2,7,9,10)$ puesto que mejoran la $\mathrm{AV}$ de estos pacientes y presentan menores riesgos que los procedimientos quirúrgicos (anillos corneales, queratoplastias).

Animamos a los contactólogos a que traten los astigmatismos irregulares advirtiéndoles que es necesario disponer de un gran abanico de modelos de lentes de contacto (11), sin obsesionarse en un único tipo, así como paciencia y perseverancia para llegar a un buen resultado funcional.

Desgraciadamente no podemos terminar aclarando ninguna receta «mágica» para adaptar lentes de contacto en este tipo de córneas. Ni siquiera podríamos intentar correlacionar las medidas refractivas preadaptación con el tipo de lente finalmente adaptada puesto que el EE preadaptación es, en la mayoría de las ocasiones, imposible de determinar (8). Nosotros seguimos después de muchos años utilizando el método de ensayo y error.

\section{BIBLIOGRAFÍA}

1. Ridley F. Contact lenses in treatment of keratoconnus. Br J Ophthalmol 1956; 40: 295-304.

2. Smiddy WE, Hamburg TR, Kracher GP, Stark WJ. Keratoconus. Contact lens or keratoplasty? Ophthalmology 1988; 95: 487-492.

3. Yeung K, Eghbali F, Weissman BA. Clinical experience with piggyback lens systems on keratoconic eyes. J Am Optom Assoc 1995; 66: 539-543.

4. Lim N, Vogt U. Characteristics and functional outcomes of 130 patients with keratoconus attending a specialist contact lens clinic. Eye 2002; 16: 54-59.

5. Maguen E, Caroline P, Rosner IR, Macy JI, Nesburn AB. The use of the Softperm lens for the correction of irregular astigmatism. CLAO 1992; 18-23: 173-176.

6. O'Donnell C, Maldonado-Codina C. A hiperDK piggyback contact lens system for keratoconus. Eye Contact Lens 2004; 30: 44-48.

7. Lass JH, Lembach RG, Park SB, Hom DL, Fritz ME, Svilar GM, et al. Clinical management of keratoconus. A multicenter analysis. Ophthalmology 1990; 97: 433-445.

8. Mehta M, Bhagwanjee A, Hilliar O. A clinical and optical evaluation of a modified lens for irregular cornea. Clin Exp Optom 2006; 89: 30-36.

9. Titiyal JS, Sinha R, Sharma N, Sreenivas V, Vajpayee RB. Contact lens rehabilitation following repaired corneal perforations. BMC Ophthalmol 2006; 14: 6:11.

10. Gruenauer-Kloevekom C, Kloevekom-Fischer U, Duncker GI. Contact lenses and special back surface design after penetrating keratoplasty to improve contact lens fit and visual outcome. Br J Ophthalmol 2005; 89: 1601-1608.

11. González-Méijome J, De Almeida JB. Parafita MA. Soft contact lenses for keratoconus: case report. Eye Contact Lens 2006; 32: 143-147. 